University of San Diego

Digital USD

Spring 5-26-2018

\title{
What's Keeping America's Heroes Awake? Screening for Obstructive Sleep Apnea in Male Veterans with Hypertension and Diabetes
}

Aaron James Stirling

University of San Diego, astirling@sandiego.edu

Follow this and additional works at: https://digital.sandiego.edu/dnp

Part of the Family Practice Nursing Commons

\section{Digital USD Citation}

Stirling, Aaron James, "What's Keeping America's Heroes Awake? Screening for Obstructive Sleep Apnea in Male Veterans with Hypertension and Diabetes" (2018). Doctor of Nursing Practice Final Manuscripts. 68.

https://digital.sandiego.edu/dnp/68

This Doctor of Nursing Practice Final Manuscript is brought to you for free and open access by the Theses and Dissertations at Digital USD. It has been accepted for inclusion in Doctor of Nursing Practice Final Manuscripts by an authorized administrator of Digital USD. For more information, please contact digital@sandiego.edu. 
UNIVERSITY OF SAN DIEGO

Hahn School of Nursing and Health Science:

Beyster Institute for Nursing Research

DOCTOR OF NURSING PRACTICE PORTFOLIO

by

Aaron James Stirling BSN, RN, PHN

A portfolio presented to the

FACULTY OF THE HAHN SCHOOL OF NURSING AND HEALTH SCIENCE UNIVERSITY OF SAN DIEGO

In partial fulfillment of the

requirements for the degree

DOCTOR OF NURSING PRACTICE

May 2018 


\section{Table of Contents}

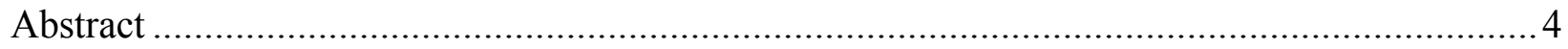

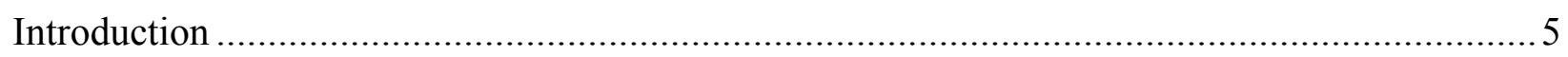

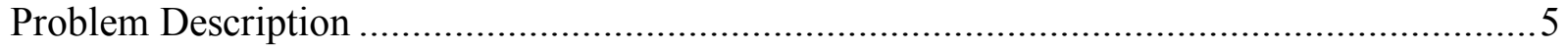

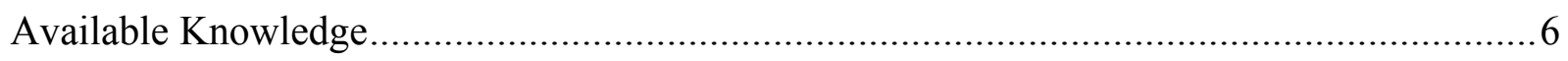

Rationale

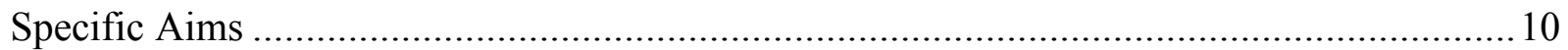

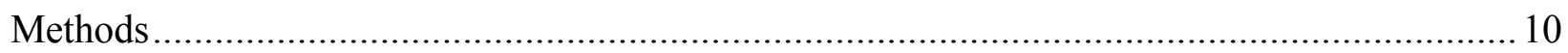

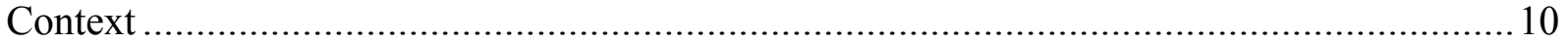

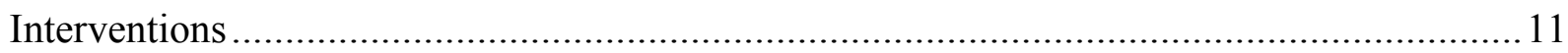

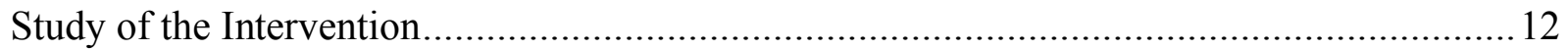

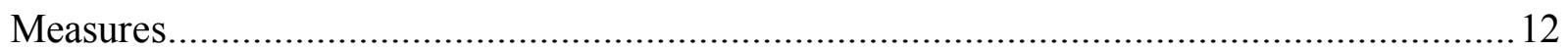

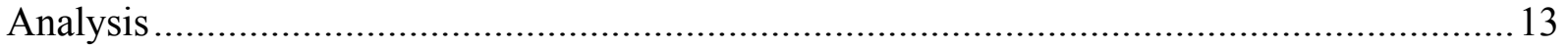

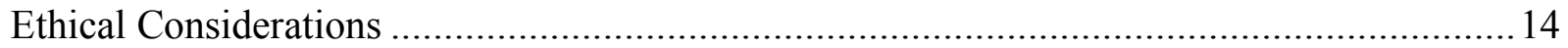

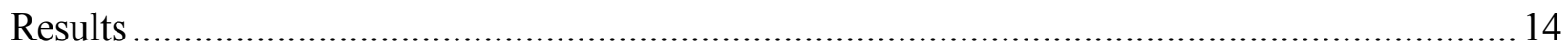

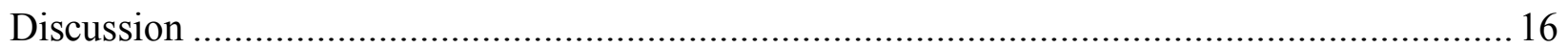

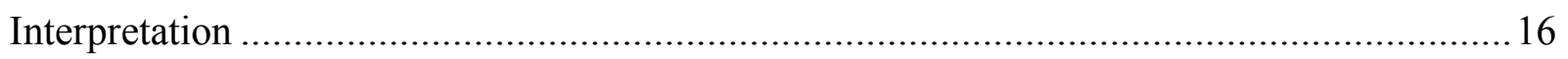

Strategies for Successful Obstructive Sleep Apnea Management .......................................... 19

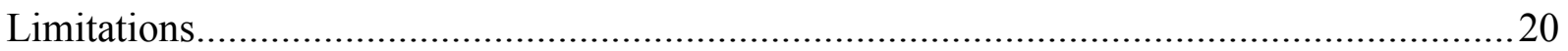

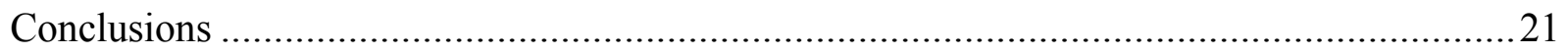

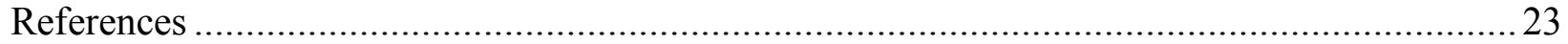

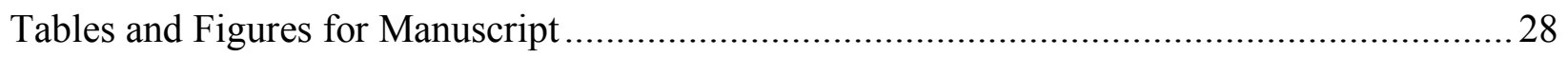




\section{What's Keeping America's Heroes Awake? \\ Screening for Obstructive Sleep Apnea in Male Veterans with Hypertension and Diabetes}

Authors: Aaron James Stirling BSN, RN, PHN; Joseph Burkard, DNSc, CRNA; David

Bittleman, MD

Author affiliations: University of San Diego, Hahn School of Nursing and Health Science:

Beyster Institute for Nursing Research (Joseph Burkard, DNSc, CRNA, and Aaron Stirling DNP

Student); Veterans Health Administration Mission Valley Clinic (David Bittleman, MD)

Corresponding author: Aaron Stirling DNP Student (760) 207- 5219,

Aaronstirling@gmail.com 


\begin{abstract}
Purpose: To reduce cardiovascular risk through optimal obstructive sleep apnea (OSA) management with proactive screening utilizing the Epworth Sleepiness Scale (ESS) in male Veterans with hypertension (HTN) and diabetes mellitus (DM).

Background: OSA is a common finding in primary care, with 1 in 5 adults feeling its effects: excessive sleepiness, impaired cognition, reduced quality of life and increased mortality. Unmanaged OSA has been highly correlated with increased cardiac risk in populations with chronic diseases such as HTN and DM. Fortunately, identification and treatment of OSA has been shown to improve cardiovascular outcomes and reduce mortality. Evidence supports outpatient screening to identify patients at risk for OSA, in order to more effectively manage future cardiovascular risk.
\end{abstract}

Strategy and Implementation: Analyzing an existing database of 146 outpatient male veterans with HTN and DM, revealed that 52\% have an existing OSA diagnosis, while the remainder have not been screened. The Doctor of Nursing Practice (DNP) student will screen these remaining patients for OSA via telephone utilizing the ESS tool. Based on findings, the DNP student will provide evidence-based guidance for further management of OSA to the care team. Evaluation/Outcomes: Post telephone screening intervention, the DNP student would expect a 40-50\% increase in completed OSA screenings by January 2018.

Implications for Practice: Implementing proactive evidence-based OSA screening is the missing link in managing HTN and DM leading to improved cardiovascular outcomes for this Veteran population.

Keywords: Obstructive sleep apnea, Epworth Sleepiness Scale, hypertension, diabetes mellitus, Veterans Health Administration, screening, primary care. Word count: 248 


\section{Introduction}

\section{Problem Description}

Obstructive sleep apnea (OSA) is a multifaceted and surprisingly common disease that occurs in one out of five adults in the United States. OSA is often underdiagnosed and subsequently undertreated due to its subtle presentation for both patients and providers (Gonzaga, Bertolami, Bertolami, Amodeo, \& Calhoun, 2015). Screening for OSA in primary care is problematic as patients do not report sleep disturbances and providers do not routinely assessing for sleep-related symptoms (Miller \& Berger, 2016). Thus, as little as 15\% of patients with clinically significant OSA are identified and diagnosed (Miller \& Berger, 2016). To further compound this issue, untreated OSA significantly contributes to the risk for developing and dying from cardiovascular disease and increased all-cause mortality (Gonzaga et al., 2015; Miller \& Berger, 2016).

OSA disturbs homeostasis by inducing intermittent hypoxemia, resulting in a surge of sympathetic activation and initiating a disruptive cascade that is evident in cardiovascular and metabolic processes. This disruption been shown to increase the risk for developing hypertension (HTN) and complicating treatment (Dempsey, Veasey, Morgan, \& O'Donnell, 2010; Tanigawa, 2011; Walia et al., 2014). Furthermore, OSA induced disruptions lead to poorer glycemic control via decreased insulin sensitivity, a higher risk for developing diabetes mellitus (DM), and more than doubles the risk of having an uncontrolled HgbA1C in diabetic patients (Muraki et al., 2010; Torrella et al., 2015).

There are 1.5 million veterans diagnosed with OSA in the United States today. These numbers are only increasing as OSA disease prevalence has doubled with the explosive 6-fold growth of this population in recent years (Bravata, Lightner, Yaggi, \& Miech, 2018; Jackson et 
al., 2017). Additionally, the veteran population is at an inherently increased risk for OSA due to the high proportion of males, increasing age, and prevalence of obesity (Bravata et al., 2018). This risk was demonstrated in an analysis of Mission Valley primary care male veterans with diagnoses of HTN and DM that revealed a majority (51\%) of this population had a concurrent diagnosis of OSA. The remaining population was potentially at risk for OSA and was recommended to be screened (Moga, Burkard, Bittleman, \& Hua Liu, 2017).

\section{Available Knowledge}

To support this evidence-based practice (EBP) screening project, a thorough literature search was completed using open access and subscription-based search engines provided through University of San Diego's Copley Library. Search engines included CINAHL Plus with Full Text, PubMed, Google Scholar, Cochrane Database of Systematic Reviews, and Ovid. Search terms and medical subject headings included sleep apnea, obstructive sleep apnea, syndromes/complications, diabetes, hypertension, Veterans Health Administration, VA, veterans, primary care, screening sleep apnea, Epworth Sleepiness Scale. Articles included were published in the English language from the year 1995 to present. The initial search returned a total of 365 articles and was narrowed based on subject content and relevance to 17. A total of 13 articles were used as evidence for the intervention. Of the articles selected, 8 were systemic reviews, 3 randomized control trials, and one controlled cohort study.

Provider assessment and screening of OSA symptoms (e.g., excessive daytime sleepiness [EDS]) are essential for diagnosis as reported by Miller and Berger (2016) in a systematic review of the available literature for screening and assessment of OSA in primary care. They reported that only $6 \%$ of patients present with sleep-related chief complaints in primary care due to the chronic and difficult-to-describe nature of the symptoms. Furthermore, they endorsed the 
American Academy of Sleep Medicine recommendations reported by Epstein et al. (2009) for routine primary care screening of OSA, especially in high risk patients with HTN and DM. Additionally, they emphasized the importance of expedited referrals to sleep medicine for timely diagnosis and treatment for stabilization of the disease and for improved cardiovascular outcomes.

Dempsey et al. (2010) provided a systematic review of the literature focusing on the pathophysiology of OSA. They noted that OSA induced transient obstruction of the upper airways, subsequent fluctuations in arterial oxygenation, and resulting intermittent hypoxemia leads to arousals during sleep with sympathetic nervous system overcorrection. They report these surges in sympathetic activation to complicate the treatment of chronic illnesses such as HTN and DM. Supporting the interrelation on OSA in patients with HTN and DM was found in the following studies. Gonzaga et al. (2015) in a systemic literature review investigated the interrelation between HTN and OSA. They discovered that $30-40 \%$ of hypertensive patients tested positive for OSA and 50\% of patients with OSA were found be hypertensive, further indicating the need to screen patients with HTN for OSA. Muraki et al. (2010) In a large cohort study, associated nocturnal intermittent hypoxia with increased risk for the development of DM type 2 independent of age, sex, and body mass index. Aurora and Punjabi (2013) in a review of the literature identified a similar independent bidirectional association of DM type 2 and OSA and emphasized the need to routinely screen for OSA in patients with DM.

The Epworth Sleepiness Scale (ESS) was designed by Johns (1991) to assess subjective, excessive daytime sleepiness (EDS) in patients. In his more recent review, Johns (2009) described EDS as a symptoms that occurs when a patient is expected to remain awake and alert during daytime activities but instead feels drowsy or falls asleep. He reports that the ESS is 
designed as a retrospective recall of the patient's dozing behavior in eight scenarios on a 0 (Never) to 3 (Always) scale and a total score from 0 to 24 points. His review of the tool literature reported normal scores $(M=4.6 ; S D=2.8)$ for populations without sleep disorders and determined that patients scoring above 10 points were recommended for further follow up. Hardinge, Pitson, and Stradling (1995) further confirmed EDS as a main presenting symptom of patients with undiagnosed OSA. For this reason, they reported the importance to routinely assess for EDS in patients with comorbidities such as HTN and DM in the primary care setting. Devine, Hakim, and Green (2005) in a systematic review of sleep dysfunction measurement tools noted the ESS as simple to use and interpret, with sufficient reliability and responsiveness, and validated in a variety of populations.

The benefits of identifying and treating OSA for patients with HTN are demonstrated in a meta-analysis by Montesi, Edwards, Malhotra, and Bakker (2012). They determined that OSA treatment with continuous positive airway pressure (CPAP) yielded significant reductions in diurnal and nocturnal systolic/diastolic blood pressures when compared with placebo and sham CPAP therapy. The researchers also noted the impact of OSA treatment on population health with reductions in daytime sleepiness, driving risk, and increased longterm cardiovascular benefits. For diabetic patients with OSA, Torrella et al. (2015) in a non-randomized control trial, demonstrated improved nocturnal and postprandial peaks of interstitial glycemia with CPAP treatment. Marin, Carrizo, Vicente, and Agusti (2005) demonstrated long term reductions in cardiovascular risk with OSA treatment via CPAP in their prospective cohort study. Recruiting and following an age- and BMI-matched cohort of healthy men with and without OSA for a period of 12 years, fatal and non-fatal cardiac events were found to have increased in patients 
with untreated severe OSA. Furthermore, they demonstrated that treatment with CPAP for at least 4 hours per night significantly reduce cardiovascular risk for those patients.

After a careful search and analysis of the literature, there was sufficient evidence to support an ESS screening intervention to proactively assess for OSA in the primary care setting for patients with HTN and DM. In addition, evidence assessed is applied to provide evidencebased recommendations for optimized disease management and cardiovascular risk aversion in OSA patients.

\section{Rationale}

The Iowa Model is scalable and well suited for implementing EBP projects in large multihospital settings as well as in small primary care clinics. The model's emphasis on assessing problem-focused triggers, organizational priority of the problem, and a sufficient evidence base will ensure the project is successful in the VA primary-care setting (Titler et al., 2001). Strengths of this model include its ease of use, application focus, and integration of the interdisciplinary team. Challenges with using this model include the potential for processes to become fragmented with built-in feedback loops and the introduction of multiple stakeholders can introduce unwanted complexities (Titler et al., 2001).

The 5 A's for Patient-centered Counseling (5As) model was utilized for the structure of this telephone screening intervention. The 5As is an evidence-based construct that was initially developed by the National Cancer Institute to assist providers in counseling for smoking cessation (Whitlock, Orleans, Pender, \& Allan, 2002). The 5As has reported successful interventions in primary care and in a variety of health conditions and behaviors (Whitlock et al., 2002). The success of the 5As lies in its simplicity and logical format representing behavior change as a cyclical pathway. The 5As pathway begins with Ask to assess patient behavior, 
beliefs, and motivation followed by Advise for patient-specific health risks, then to Agree on a realistic set of goals, Assist with anticipated barriers, and finally to develop an action plan and Arrange follow-up support (Whitlock et al., 2002). With the evidence-based structure provided by the Iowa Model and guided by the 5As, the screening intervention was successful and provided important OSA screening to a high-risk population of veterans.

\section{Specific Aims}

The primary outcome for this intervention was to increase screening for the remaining 70 primary-care, male veterans with HTN and DM but without OSA. The short-term goal (30 days) was to screen $40 \%-50 \%$ of this group with the ESS by January 2018 . The long-term goal ( 90 days) was to provide evidence-based guidance to the care team for further management of OSA.

\section{Methods}

\section{Context}

The VA is the largest integrated health care system in the United States, with over 12,000 health care facilities, 170 hospitals and approximately 1,000 outpatient clinics (U.S. Department of Veterans Affairs, 2018). The VA health care system spans coast-to-coast and provides the entire continuum of care for over 9 million Veterans (U.S. Department of Veterans Affairs, 2018). The local VA Mission Valley primary care clinic was selected for implementing this EBP screening intervention due to physician and care team engagement, site willingness, and experience with EBP projects. The database used in this intervention was generated from the VA's electronic health record (EHR) for Moga et al. (2017)'s DNP Project, "Evaluation of Hypertensive Clinical Significance in a Primary Care Setting in Veterans with Diabetes". This dataset was further refined to male patients with diagnoses of HTN and DM without OSA. This 
sub-group of patients was overall described as older, overweight, and with controlled HTN and DM (Tables 1 and 2).

Potential project challenges due to contextual factors included the complexity and size of the VA Health Care system as whole as well as the integration of the interdisciplinary team for follow up to ensure ESS scores were assessed and referred as appropriate.

\section{Interventions}

For this intervention, the DNP student functioned as a phone screener, data collector, and analyst. The care team, composed of the primary care physician and registered nurses, functioned in support roles and were the primary stakeholders in this project. The project implementation was divided into three stages: (a) in the first stage, the dataset used by the preceding DNP student was refined for the current project; (b) the second stage incorporated the telephone screening intervention, and (c) the third stage was analysis and reporting back to the care team.

Stage 1 began in August 2017 by first refining and de-identifying the existing database of 331 patients to focus on male patients with documented HTN and DM diagnoses. The database was further refined for patients without a diagnosis of OSA (Figure 1). The remaining 70 patients eligible for screening were cross-referenced in the VA's EHR for contact information. A total of 14 patients were excluded from the intervention due to non-working phone numbers or inaccessible charts flagged as "sensitive" in the EHR. Stage 2 began in late October 2017 by screening the remaining 56 eligible patients via phone with a standardized script incorporating the ESS and the 5As (Figure 2). Screening with the ESS produced a score of 0 to 24 for each patient and was recorded at the conclusion of the call. Missed calls were logged and voicemail messages were left with a callback number and time for a follow-up call by the DNP student. Any additional needs or concerns voiced by the patient during the screening intervention were 
added to the log and reported back to the care team. For more urgent needs, the DNP student referred the patient to the clinic's main number for more timely follow up. Stage 2 was concluded after 30 days of data collection with satisfactory screening rates exceeding projected goals.

In the third and final stage of the intervention, the collected data were analyzed and results were presented to the stakeholders in mid-December 2017. Results were disseminated at two professional conferences in November 2017 and March 2018. Furthermore, the discussion section of this manuscript will provide further guidance to the care team regarding evidencebased management of this high-risk population.

\section{Study of the Intervention}

For the intervention to be successful and the findings valid, it was important to assess and control for contextual factors at the VA Mission Valley clinic. For example, ensuring that there were no changes in the sleep health related standard of care, or concurrent projects during the intervention period offered creditability that the observed increase in screening could be directly attributed to the work done by this project.

\section{Measures}

To ensure data integrity and the ability to draw conclusions from the intervention, process monitoring was fundamental. To eliminate possible process fragmentation, the DNP student was responsible for database abstraction, patient screening, and data entry. The process of identifying patients in the database who met criteria for screening as well as pairing with contact information was important to complete early in the project to proceed with the intervention. During this process, to mitigate fatigue, database abstraction was divided over 2 days and processed in batches to ensure data quality and accuracy. This process was assessed initially and then weekly 
for quality and discrepancies during data collection. Intervention call volume was high and accurate data entry proved to be an ongoing challenge. To assist with this process, collected data was entered immediately after screening and was audited for errors at the end of each working day. Finally, measurement of the overall ESS screening percentage was audited at the end of each week to ensure consistent screening rates and satisfactory progress towards project outcomes.

\section{Analysis}

The initial statistical analysis utilized R (Version 3.1.1) with an overlay of Radiant (Version 0.527) to run a linear regression analysis to assess for correlations with ESS score totals and continuous dataset demographics (e.g., blood pressure, A1C, disability rating). This analysis allowed the DNP student to visualize positive and negative correlations within the dataset to investigate for further analysis. Further regression analysis was performed with Excel Analysis ToolPak (Version 16.10) software providing item-specific statistical output.

Establishing the risk profile of the screened patients was performed by calculating the mean ESS total for comparison to normalized populations and is displayed visually on a Box Plot and table (Figure 3, Table 3). Furthermore, to visually demonstrate EDS related risk at the individual level the ESS total score for each patient was arranged in ascending order and classified with corresponding EDS risk categories and displayed in a Bar Chart and table (Figure 4, Table 4). Additionally, analysis of item responses was performed on patient scoring tendencies of the ESS and displayed in a frequency table (Table 5). Call volumes and screening attempts during the intervention were analyzed and shown in a table (Table 6). Variations within the data could be attributed to the small sample and scope of this intervention. Likewise, the dataset 
provided a single data point and did not allow for trending of data or historical comparisons of the data collected.

\section{Ethical Considerations}

This retrospective data analysis and screening intervention was approved by the Institutional Review Boards at both the Department of Veterans Affairs and the University of San Diego as an extension of the previous DNP projects H160114 and 2016-12-107, respectively. Permission for the use of the ESS tool was acquired from the Mapi Research Trust (Reference 126377) at no cost due to the unfunded academic nature of this project. Additionally, the author did not have any conflicts of interest to disclose with this project.

\section{Results}

The screening intervention was successful with 47 (84\%) of the 56 identified male patients with HTN, DM and without OSA were screened utilizing the ESS within the 30-day timeline; surpassing the goal of screening at least $50 \%$ of the population by January 2018 (Figure 1).

Important changes to the intervention occurred in Stage 2 when the DNP student discovered that the clinic phone to be used to screen patients was unable to receive direct calls. To address this challenge, a VoIP account was added to the DNP student's phone that allowed for a consistent phone number for patients to return missed screening calls. This process change was essential as patients could return calls directly to the DNP student, ensuring consistent follow up, and facilitating the success of the intervention.

The average total of ESS scores for the screened population $(M=7.34 ; S D=4.4)$ indicate an elevated EDS and an increased risk for OSA when compared to normalized populations (Figure 3, Table 3). Moreover, the high-risk characteristics of the screened population discovered 
at baseline were reinforced by the findings of increased age $(M=67 ; S D=9.8)$, overweight $\left(M_{\mathrm{BMI}}=29.61 ; S D=4.56\right)$, along with a high prevalence of dyslipidemia $(83 \%)$, chronic kidney disease $(28 \%)$, and coronary artery disease (19\%).

Table 4 demonstrates the EDS risk stratification for the population screened with the ESS. Based on the outlined EDS categories, $40 \%$ of the population exhibited higher than normal daytime sleepiness with scores ranging from 6 to 10. Furthermore, 24\% of the population exhibited mild to severe excessive daytime sleepiness with scores ranging from 11 to 24. (Figure 4).

Table 5 illustrates the distribution of patient scores for each of the eight scenarios. Scenarios with dramatic skews of scoring to "0" or "would never doze" were seen in Questions 4,6 , and 8 with $66 \%, 91 \%$, and $89 \%$ of the patients, suggesting this response indicated activities that respondents believed required more alertness. Items that were more focused on home, lying down to rest or after a meal were found to have an even distribution of scoring among respondents.

In a multiple regression equation with the ESS score as the dependent variable, the only significant independent variable was the patient's VA assigned disability rating $(F=5.863, p=$ $.020)$. The correlation of ESS and disability rating was small (adj $\left.R^{2}=0.096\right)$ but suggests further investigation may be warranted (Table 7).

For this intervention, a total of 84 calls were made to 56 patients and screened with the ESS tool. At the conclusion of the intervention, a total of 47 (84\%) patients were screened with a total of 66 calls, averaging 1.4 calls for each completed screening. For each screening call that connected the DNP student with a patient, the ESS was successfully completed $100 \%$ of the time (Table 6). 
The remaining $9(16 \%)$ patients that were not screened during the intervention could not be successfully reached via phone after numerous attempts. Patients received two follow up calls over the intervention period and, with each missed call, a voice mail was left for a total of 18 calls. Of the unscreened patients, no call-backs to the DNP student were made during the intervention timeframe (Table 6).

\section{Discussion}

Screening veterans with HTN and DM at risk for undiagnosed OSA with the ESS was successful with $84 \%$ of the target population screened within the intervention timeline. Important findings included the increased average ESS total of this group $(M=7.4 ; S D=4.4)$ when compared with other normalized populations $(M=4.28 ; S D=.45)$ throughout the world. Applying the EDS classifications finds that $40 \%$ of the population was categorized with higher than normal daytime sleepiness (i.e., ESS score from 6-10) and 24\% of the population exhibited abnormal amounts of EDS (i.e., ESS score from 11-24).

Strengths of this intervention include a focus on implementation in primary care setting where providers are more likely to encounter patients with undiagnosed OSA (Miller \& Berger, 2016). Furthermore, this intervention explored the important interrelation between the diagnoses of HTN, DM, and OSA for improved disease management and cardiac risk aversion.

\section{Interpretation}

The elevation of the screened population's total ESS scores when compared to populations without sleep disorders demonstrated an increased risk for undiagnosed OSA. To compound this risk, veteran OSA epidemiological studies have correlated higher total ESS scores with increased severity of OSA and subsequent increase of cardiovascular and metabolic comorbidities (Samson, Casey, Knepler, \& Panos, 2012). Given the established baseline elevated 
OSA risk for veterans as a population, the increased ESS scores collected places this population at considerable risk for undiagnosed OSA. This further reinforces the need to routinely screen patients with HTN and DM for OSA in the primary care setting.

The low rates of assessment and diagnosis of OSA in primary care reported in other studies is similarly reflected in the screened population (Miller \& Berger, 2016). Based on the recommended 10-point cutoff for a normal ESS score, nearly one-fourth of the screened population demonstrated an abnormal amount of EDS and without routine screening, these higher risk EDS groups would be overlooked. The remaining $40 \%$ of the population classified on the upper-end of normal daytime sleepiness (ESS 6-10) and while not scoring abnormally, warrants closer observation and clinical management by the care team.

Analysis of the scoring tendencies of the screened population demonstrated the intentional design of the ESS to represent situations with varying chance of dozing. Situations requiring more alertness, such as the Question 6 scenario, "talking with someone," or Question 8, in a car ... in traffic," were scored in a corresponding manner by patients (Kendzerska, Smith, Brignardello-Petersen, Leung, \& Tomlinson, 2014). Similarly, the Question 5 scenario, "lying down to rest," required less alertness and was scored as such by many patients (Table 5). The author of the tool notes that all questions must be completed for the screening to be valid, thus the tool was designed to be flexible and to allow interpretation for a variety of patients (Johns, 2009). This is seen in Questions 4 and 8, where the patient was asked to remember a time where they were "a passenger in a car" versus "in a car" in this manner the tool deliberately does not identify if the patient was the driver or a passenger and allowed patients who do not drive to complete the screening. The adaptability of the ESS permitted increased screening success in a diverse patient population, especially in older veterans who may no longer drive. 
The statistical significance of a positive correlation in ESS total scores and the VA recorded disability rating could be attributed to the retrospective and subjective nature of the ESS scale. The ESS relied on the patient's recall of potentially dozing in everyday situations and could overlap with the patient's overall appraisal of their quality of life. Studies have shown that veterans reporting higher EDS also report lower perceived quality of life, this is especially evident in populations with sleep disorders in combination with chronic diseases (Alexander et al., 2016; Vinnikov, Blanc, Alilin, Zutler, \& Holty, 2017). Thus, increasing EDS measured with the ESS in this intervention could likely be positively correlated to the increased disability rating and subsequent lower perceived quality of life in the screened veterans.

Screening interventions via phone can be challenging. Contributing to the success of the intervention was the engagement of this veteran population. A unique theme observed during screening calls was the willingness to participate, not only for their own benefit, but also for the benefit of other veterans. This was demonstrated by the $100 \%$ screen completion of the calls answered or returned to the DNP student and the low amount of calls required to reach patients for screening (Table 7). Perhaps this willingness to participate derived from the cohesion of veterans as a population and greatly contributed to the success of this screening intervention. Assessing the cost to benefit analysis for this intervention was challenging as the total costs and potential savings of OSA treatment are not always directly measurable. Overall intervention cost was minimal (Figure 5), only requiring labor hours of the screener and 1 hour of training. Moreover, the ESS tool was used at no cost as it is integrated into the VA EHR. Potential cost savings were estimated by calculating the cost of OSA related hospitalizations associated with HTN (2.1\%) and DM (2.9\%) and were extrapolated to the veteran population of 7\% the United States (Leger, Bayon, Laaban, \& Philip, 2012; National Center for Veterans 
Analysis and Statistics, 2016). Overall, this intervention provides an estimated savings of $\$ 7.80$ initially and $\$ 11$ to sustain for each dollar spent (Figure 6). This low cost and high benefit to the VA is attributed to the proactive nature of this screening intervention, to mitigate future costs by identifying OSA patients in primary care with HTN and DM.

\section{Strategies for Successful Obstructive Sleep Apnea Management}

The final endpoint of this screening intervention was to provide the care team with evidence-based recommendations for the management of this population. Utilizing the current American Academy of Sleep Medicine guidelines for management of OSA in adults, CPAP is considered the most effective treatment to provide to splint open the upper airway to reduce the number of hypoxemic events during sleep (Epstein et al., 2009). Other alternative interventions to treat OSA are custom oral appliances that assist in maintaining airway patency, surgery for upper airway reconstruction, or behavioral changes including weight loss to a BMI less than 25, sleep positioning, and reducing or avoiding alcohol and sedatives before sleeping (Epstein et al., 2009).

Consistent CPAP therapy in patients with HTN has been demonstrated to significantly reduce both SBP and DBP when compared to other treatments modalities (Montesi et al., 2012). In addition, CPAP therapy has been shown to improve insulin sensitivity in patients starting at $18 \%$ with 2 days of treatment and increasing to $31 \%$ after 3 months of treatment and with positive effects lasting for years in compliant patients (Dempsey et al., 2010). Moreover, CPAP treatment significantly reduces the risk for fatal and non-fatal cardiovascular events in patients with severe OSA, effectively lowering their risk to that of healthy individuals (Marin et al., 2005). 
The positive impact of CPAP on OSA and subsequent comorbidity is reliant on patient compliance and duration of the treatment. Reduction in cardiovascular risk has been shown with CPAP use for at least 4 hours per night (Marin et al., 2005). Veteran epidemiological studies have indicated that there is a challenge in compliance with CPAP treatment. Large scale studies have shown that a little over half of veterans were compliant with their prescribed CPAP regimen for more than three nights weekly (Samson et al., 2012). It is important for the care team to recognize the benefits of forming a realistic plan of care that meets the needs of patients and care providers and is realistic and achievable.

A patient centered approach is essential to promote CPAP adherence through patient engagement and education and is vital to treat OSA effectively (Hilbert \& Yaggi, 2017). The emphasis is placed on partnering with the patient regarding the disease prevention and health promoting aspects of CPAP therapy. With a greater understanding of the benefits of CPAP the patient is able to make more informed choices in their care, resulting in greater compliance. Moreover combining educational interventions with family/partners support have shown to increase CPAP use by at least 30 minutes a night and increased overall adherence (at least 4 hours a night) by $13 \%$ (Hilbert \& Yaggi, 2017). Collaborating with the multidisciplinary team beyond the sleep lab is also recommended. Primary care providers are an essential link in identifying OSA risk factors, abnormal clinical findings and referring appropriately and are crucial to disease prevention and risk management in this population.

\section{Limitations}

Important limitations for this intervention can be attributed to the VA database utilized. The database was inherently outdated as it was single data point in the patient's care continuum 
when queried in 2017. This limitation was expected when working with a retrospective database and has the potential to limit the generalizability of the findings.

Furthermore, the internal validity of the intervention could be limited by the absence of a validated, process measure to qualify success beyond the percentage of the total intervention population screened. The overall design of the intervention allowed for potential bias with a single DNP student screening, collecting, recording, and analyzing data. To mitigate this potential bias, the intervention was designed with multiple redundancies in the process and outcome measures, with an emphasis on the structure of the intervention and with close monitoring of the anticipated outcomes.

\section{Conclusions}

At the conclusion of this screening intervention, the DNP student was able to demonstrate an integrated, cost-effective method to proactively screen for OSA in primary care. This screening intervention identified a male veteran population with HTN and DM who was at elevated risk for OSA at baseline and, when compared, to normal populations utilizing the ESS. To assist with disease management for the population, recommendations for OSA treatment with CPAP could minimize disturbances and improve HTN, DM, and cardiovascular outcomes. Additionally, delivering care in a patient-centered approach improved adherence to CPAP treatment and ultimately long-term health outcomes for OSA.

Based on the minimal cost of the intervention and the predicted benefits to population health, this intervention is expected to be sustainable. There is also the potential for a future DNP student to assess the impact of CPAP treatment over time on the screened population via serial ESS scores and with a health-related quality of life tool. Dissemination of this intervention to 
similar VA primary care clinics is feasible due to the integrated and scalable nature of the VA health system infrastructure.

Further study is needed on the interplay of OSA and comorbid conditions such as HTN and DM in controlled and demographically-matched populations to further reinforce the benefit of OSA treatment. In addition, further investigation into the cost of OSA at the population level may allow for a more accurate financial analysis of the downstream impact of untreated OSA. In addition to its stated screening goals, the intervention succeeded empowering the routine screening for OSA in this veteran population and more importantly to ask our heroes "What's keeping you awake?" 


\section{References}

Alexander, M., Ray, M. A., Hebert, J. R., Youngstedt, S. D., Zhang, H., Steck, S. E., .. . Burch, J. B. (2016). The national veteran sleep disorder study: Descriptive epidemiology and secular trends, 2000-2010. Sleep, 39(7), 1399-1410. doi:10.5665/sleep.5972

Aurora, R. N., \& Punjabi, N. M. (2013). Obstructive sleep apnoea and type 2 diabetes mellitus: A bidirectional association. The Lancet Respiratory Medicine, 1(4), 329-338. doi:http://dx.doi.org/10.1016/S2213-2600(13)70039-0

Bravata, D. M., Lightner, N., Yaggi, H. K., \& Miech, E. J. (2018). Economic assessment of 4 approaches to the diagnosis and initial treatment of sleep apnea. Respiratory Care, 63(1), 50-61. doi:10.4187/respcare.05355

Dempsey, J. A., Veasey, S. C., Morgan, B. J., \& O'Donnell, C. P. (2010). Pathophysiology of sleep apnea. Physiological Reviews, 90(1), 47-112. doi:10.1152/physrev.00043.2008

Devine, E. B., Hakim, Z., \& Green, J. (2005). A systematic review of patient-reported outcome instruments measuring sleep dysfunction in adults. Pharmacoeconomics, 23(9), 889-912. doi:10.2165/00019053-200523090-00003

Epstein, L. J., Kristo, D., Patrick J. Strollo, J., Friedman, N., Malhotra, A., Patil, S. P., . . . Weinstein, M. D. (2009). Clinical guideline for the evaluation, management and longterm care of obstructive sleep apnea in adults. Journal of Clinical Sleep Medicine, 5(3), 263-276. Retrieved from http://www.ncbi.nlm.nih.gov/pmc/articles/PMC2699173/

Gonzaga, C., Bertolami, A., Bertolami, M., Amodeo, C., \& Calhoun, D. (2015). Obstructive sleep apnea, hypertension and cardiovascular diseases. Journal of Human Hypertension, 29(12), 705-712. doi:10.1038/jhh.2015.15 
Hardinge, F. M., Pitson, D. J., \& Stradling, J. R. (1995). Use of the epworth sleepiness scale to demonstrate response to treatment with nasal continuous positive airways pressure in patients with obstructive sleep apnoea. Respiratory Medicine(89), 617-620.

Hilbert, J., \& Yaggi, H. K. (2017). Patient-centered care in obstructive sleep apnea: A vision for the future. Sleep Medicine Reviews. doi:10.1016/j.smrv.2017.02.004

Jackson, M., Becerra, B. J., Marmolejo, C., Avina, R. M., Henley, N., \& Becerra, M. B. (2017). Prevalence and correlates of sleep apnea among US male veterans, 2005-2014. Preventing Chronic Disease, 14, 1-5. doi:10.5888/pcd14.160365

Johns, M. W. (1991). A new method for measuring daytime sleepiness: The epworth sleepiness scale. Sleep, 14(6), 540-545.

Johns, M. W. (2009). What is excessive daytime sleepiness? In P. Fulke \& S. Vaughan (Eds.), Sleep deprivation: Causes, effects and treatment (pp. 1-37): Nova Science Publishers inc.

Kendzerska, T. B., Smith, P. M., Brignardello-Petersen, R., Leung, R. S., \& Tomlinson, G. A. (2014). Evaluation of the measurement properties of the epworth sleepiness scale: A systematic review. Sleep Medicine Reviews, 18(4), 321-331.

doi:10.1016/j.smrv.2013.08.002

Leger, D., Bayon, V., Laaban, J. P., \& Philip, P. (2012). Impact of sleep apnea on economics. Sleep Medicine Reviews, 16(5), 455-462. doi:10.1016/j.smrv.2011.10.001

Manni, R., Politini, L., Ratti, M. T., \& Tartara, A. (1999). Sleepiness in obstructive sleep apnea syndrome and simple snoring evaluated by the epworth sleepiness scale. Journal of European Sleep Research Society, 8, 319-320.

Marin, J. M., Carrizo, S. J., Vicente, E., \& Agusti, A. G. N. (2005). Long-term cardiovascular outcomes in men with obstructive sleep apnoea-hypopnoea with or without treatment 
with continuous positive airway pressure: An observational study. Lancet, 365(9464), 1046-1053. Retrieved from http://0-

search.ebscohost.com.sally.sandiego.edu/login.aspx?direct=true\&db=hch\&AN=1648999 $3 \&$ site $=$ ehost-live

Miller, J. N., \& Berger, A. M. (2016). Screening and assessment for obstructive sleep apnea in primary care. Sleep Medicine Reviews, 29, 41-51. doi:10.1016/j.smrv.2015.09.005

Moga, C., Burkard, J., Bittleman, D., \& Hua Liu, J. (2017). Evaluation of hypertensive clinical significance in a primary care setting in veterans with diabetes. Doctor of Nursing Practice Final Manuscripts(55). Retrieved from http://digital.sandiego.edu/dnp/55

Montesi, S. B., Edwards, B. A., Malhotra, A., \& Bakker, J. P. (2012). The effect of continuous positive airway pressure treatment on blood pressure: A systematic review and metaanalysis of randomized controlled trials. Journal of Clinical Sleep Medicine, 8(5), 587596. doi: $10.5664 /$ jcsm. 2170

Muraki, I., Tanigawa, T., Yamagishi, K., Sakurai, S., Ohira, T., Imano, H., . . Investigators, C. (2010). Nocturnal intermittent hypoxia and the development of type 2 diabetes: The circulatory risk in communities study (circs). Diabetologia, 53(3), 481-488. doi:10.1007/s00125-009-1616-0

National Center for Veterans Analysis and Statistics. (2016). California state summary. Retrieved from https://www.data.va.gov/dataset/state-summary-california

Parkes, J. D., Chen, S. Y., Clift, S. J., Dahlitz, M. J., \& Dunn, G. (1998). The clinical diagnosis of the narcoleptic syndrome. Journal of Sleep Research, 7(1), 41-52.

Samson, P., Casey, K. R., Knepler, J., \& Panos, R. J. (2012). Clinical characteristics, comorbidities, and response to treatment of veterans with obstructive sleep apnea, 
cincinnati veterans affairs medical center, 2005-2007. Preventing Chronic Disease. doi:10.5888/pcd9.110117

Tanigawa, T. (2011). Obstructive sleep apnea: Its prevention and screening may contribute to the prevention of hypertension, diabetes and cardiovascular diseases. EPMA Journal, 2(1), 83-89. doi:10.1007/s13167-011-0073-2

Titler, M. G., Kleiber, C., Steelman, V. J., Rakel, B. A., Budreau, G., Everett, L. Q., . . Goode, C. J. (2001). The iowa model of evidence-based practice to promote quality care. Critical Care Nursing Clinics of North America, 13(4), 497-509.

Torrella, M., Castells, I., Gimenez-Perez, G., Recasens, A., Miquel, M., Simo, O., .. . Sampol, G. (2015). Intermittent hypoxia is an independent marker of poorer glycaemic control in patients with uncontrolled type 2 diabetes. Diabetes and Metabolism, 41(4), 312-318. doi:10.1016/j.diabet.2015.01.002

U.S. Department of Veterans Affairs. (2018, March). About the veterans health administration. Retrieved from https://www.va.gov/health/aboutVHA.asp

Vinnikov, D., Blanc, P. D., Alilin, A., Zutler, M., \& Holty, J. C. (2017). Fatigue and sleepiness determine respiratory quality of life among veterans evaluated for sleep apnea. Health Qual Life Outcomes, 15(1), 48. doi:10.1186/s12955-017-0624-x

Walia, H. K., Li, H., Rueschman, M., Bhatt, D. L., Patel, S. R., Quan, S. F., . . Mehra, R. (2014). Association of severe obstructive sleep apnea and elevated blood pressure despite antihypertensive medication use. Journal of Clinical Sleep Medicine, 10(8), 835-843. doi: $10.5664 /$ jcsm. 3946 
Whitlock, E. P., Orleans, C. T., Pender, N., \& Allan, J. (2002). Evaluating primary care behavioral counseling interventions: An evidence-based approach 1. American Journal of Preventive Medicine, 22(4), 267-284. 


\section{Tables and Figures for Manuscript}

Table 1

Demographic Measures of Screened Patients $(n=47)$

\begin{tabular}{lcc}
\hline & $M$ & $S D$ \\
\hline Age (years) & 67.04 & 9.88 \\
Body-mass index $\left(\mathrm{kg} / \mathrm{m}^{2}\right)$ & 29.61 & 4.56 \\
VA Assigned Disability Rating (\%) & 31.91 & 38.82 \\
Systolic Blood Pressure (mm $\mathrm{Hg})$ & 126.57 & 15.30 \\
Diastolic Blood Pressure in $(\mathrm{mm} \mathrm{Hg})$ & 73.85 & 13.06 \\
Hemoglobin A1C (\%) & 6.86 & 1.19 \\
\hline
\end{tabular}

Table 2

Proportion of Co-morbidities in Screened Patients

$\%$

Current tobacco use - Smoked/Chewed (\%)

13

Chronic Kidney Disease (\%)

28

Transient Ischemia/Cerebrovascular Attack (\%)

9

Congestive Heart Failure (\%)

6

Coronary Artery Disease (\%)

19

Dyslipidemia (\%)

83

Obstructive Sleep Apnea (\%)

0

Hypertension (\%)

100

Diabetes (\%)

100 
Table 3

ESS Scoring Totals of Screened and Normal Populations

\begin{tabular}{lccc}
\hline & $M$ & $S D$ & $n$ \\
\hline VA Population Screened & 7.4 & 4.4 & 47 \\
UK & 4.5 & 3.3 & 188 \\
Italy & 4.4 & 2.8 & 54 \\
Turkey & 3.6 & 3.0 & 60 \\
Australia & 4.6 & 2.8 & 72 \\
\hline
\end{tabular}

Note. Normal population information from Izci et al. (2008); Johns and Hocking (1997);

Manni, Politini, Ratti, and Tartara (1999), Parkes, Chen, Clift, Dahlitz, and Dunn (1998). 
Table 4

ESS Score EDS Risk Stratification $(n=47)$

\begin{tabular}{lcc}
\hline & $n$ & $\%$ \\
\hline Lower Normal Daytime & 17 & 36
\end{tabular}

Sleepiness (0-5)

$\begin{array}{ll}\text { Higher Normal Daytime } & 19\end{array}$

Sleepiness (6-10)

Mild Excessive Daytime $\quad 8 \quad 17$

Sleepiness (11-12)

Moderate Excessive Daytime $\quad 1 \quad 1$

Sleepiness (13-15)

Severe Excessive Daytime $\quad 2 \quad 6$

Sleepiness (16-24)

Total

$47 \quad 100$ 
Table 5

ESS Item Responses $(n=47)$

$\begin{array}{cccc}\text { No } & \text { Slight } & \text { Moderate } & \text { High } \\ (0) & (1) & (2) & (3)\end{array}$

1. Sitting and reading

15

12

11

9

2. Watching TV

13

15

12

7

3. Sitting, inactive in a public

24

13

7

3

place (e.g. a theater or a

meeting)

4. As a passenger in a car for an

31

4

6

6

hour without a break

5. Lying down to rest in the

9

11

10

17

afternoon when circumstances

permit

6. Sitting and talking to someone

43

4

0

0

7. Sitting quietly after a lunch

14

10

17

6

without alcohol

8. In a car or bus, while stopped

42

4

1

0 
Table 6

Communication Attempts in Screened and Non-Screened Patients

\begin{tabular}{lccc}
\hline & $\begin{array}{c}\text { Screened } \\
(n=47)\end{array}$ & $\begin{array}{c}\text { Non-Screened } \\
(n=9)\end{array}$ & $\begin{array}{c}\text { Total Population } \\
(n=56)\end{array}$ \\
\hline Calls (Outgoing) & 51 & 18 & 69 \\
Calls (Incoming) & 15 & 0 & 15 \\
Calls per completed Screen & 1.4 & 0 & 84 \\
Answer to Screening Completion (\%) & 100 & 0 & 84 \\
Total Calls & 66 & 18 & \\
\hline
\end{tabular}


Table 7

Disability Rating Correlation with Total ESS Scores

\begin{tabular}{lr}
\hline \multicolumn{2}{c}{ Regression Statistics } \\
\hline Multiple R & 0.339510924 \\
R Square & 0.115267668 \\
Adjusted R Square & 0.095606949 \\
Standard Error & 4.180965236 \\
Observations & 47 \\
\hline
\end{tabular}

\begin{tabular}{lrrrrr} 
ANOVA & \multicolumn{7}{c}{ SS } & MS & \multicolumn{1}{c}{ S } & Significance F \\
\hline Regression & $d f$ & & \multicolumn{1}{c}{ SS } \\
Residual & 1 & 102.4852192 & 102.4852192 & 5.862841069 & 0.019558368 \\
Total & 45 & 786.6211638 & 17.48047031 & & \\
\hline
\end{tabular}

\begin{tabular}{lcrrrrrrr}
\hline & Coefficients & Standard Error & $t$ Stat & P-value & Lower 95\% & Upper 95\% & Lower 95.0\% & Upper 95.0\% \\
\hline Intercept & 6.155904739 & 0.792936051 & 7.763431525 & $7.63331 \mathrm{E}-10$ & 4.55884955 & 7.752959927 & 4.55884955 & 7.752959927 \\
Disability Rating & 0.038448318 & 0.015879005 & 2.421330434 & 0.019558368 & 0.00646636 & 0.070430276 & 0.00646636 & 0.070430276 \\
\hline
\end{tabular}




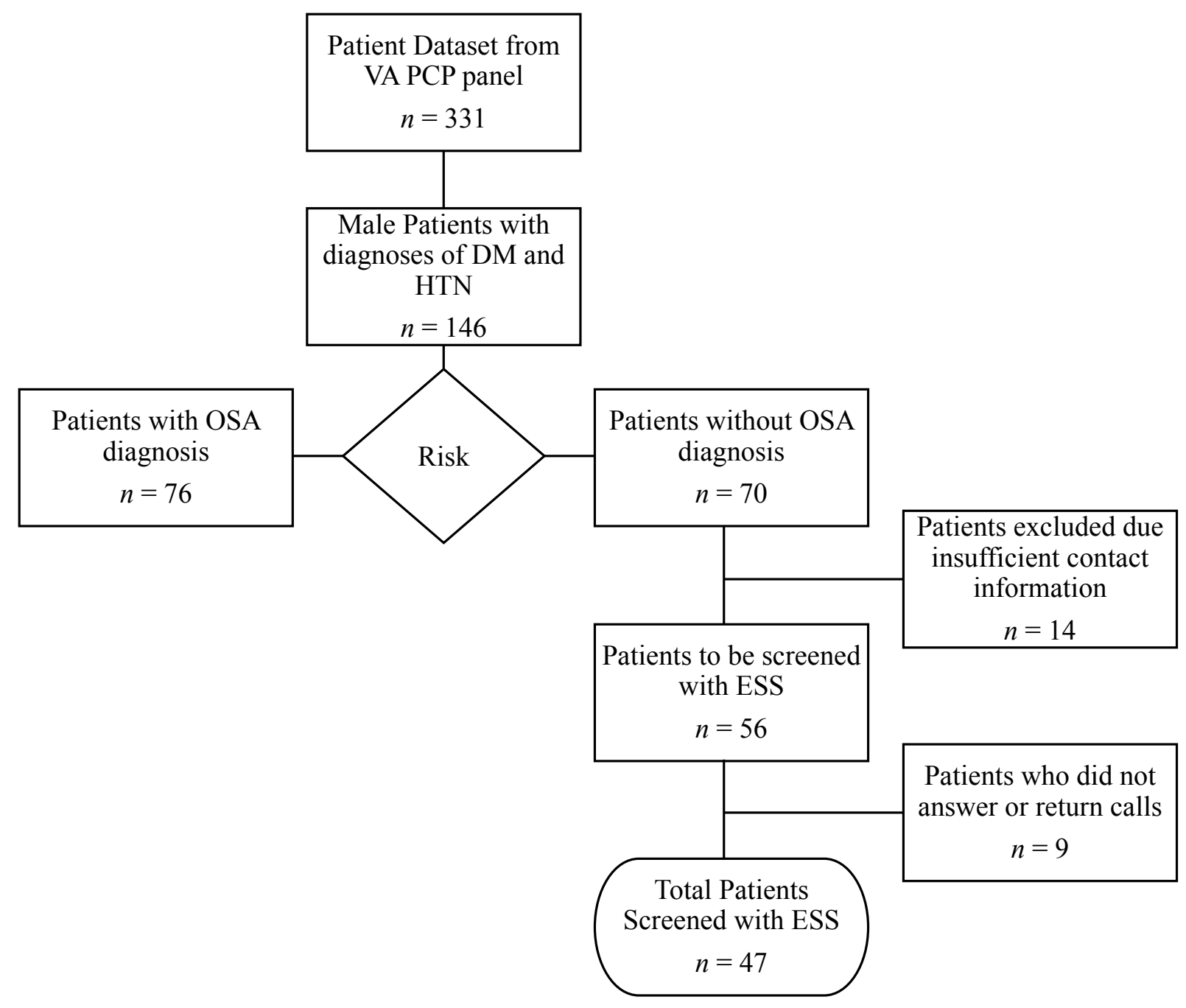

Figure 1. Project process 


\section{Phone Intervention Screening Script}

5 A's Behavior Change Model and the Epworth Sleepiness Scale

Ask:

Hello my name is Aaron, I am a nurse calling from the VA Mission Valley and we are updating our patient data base and I would like to ask you a few questions about how tired you become in your day to day life activities.

\section{Assess:}

This screening is important to identify underlying obstructive sleep apnea. Treating obstructive sleep apnea can improve your health and management of diseases such as hypertension and diabetes.

Screen with Epworth Sleepiness Scale via VA EHR.

*The questionnaire contact information and permission to use: Mapi Research Trust, Lyon,

France -Internet: https://eprovide.mapi-trust.org

Advise:

Research indicates that treating conditions such as hypertension and diabetes can be complicated with obstructive sleep apnea. Just by treating obstructive sleep apnea we can reduce blood pressure and decrease insulin resistance and allow you to live a healthier life.

Agree:

(Based on results) Formulate a care plan with the patient and with active listening, answer any questions about screening and results.

Interpretation:

0-5 Lower Normal Daytime Sleepiness

6-10 Higher Normal Daytime Sleepiness

11-12 Mild Excessive Daytime Sleepiness

13-15 Moderate Excessive Daytime Sleepiness

16-24 Severe Excessive Daytime Sleepiness

\section{Arrange:}

(Based on results) Follow up with the care team with ESS results and any patient concerns/questions from phone interview for possible sleep study referral and therapy.

Figure 2. Intervention screening script with the 5 As and the ESS 


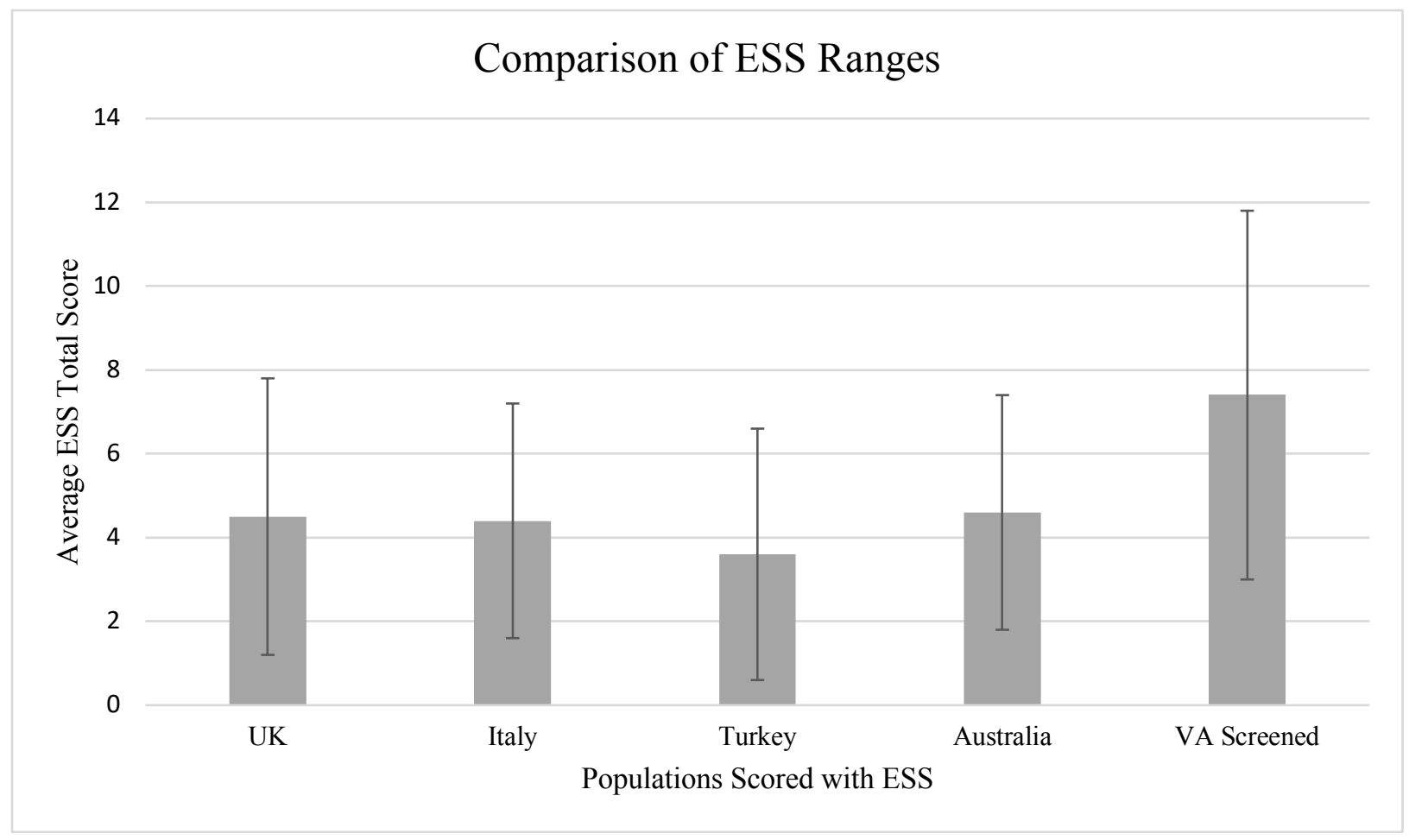

Figure 3. Comparison of normal ESS ranges and screened population 


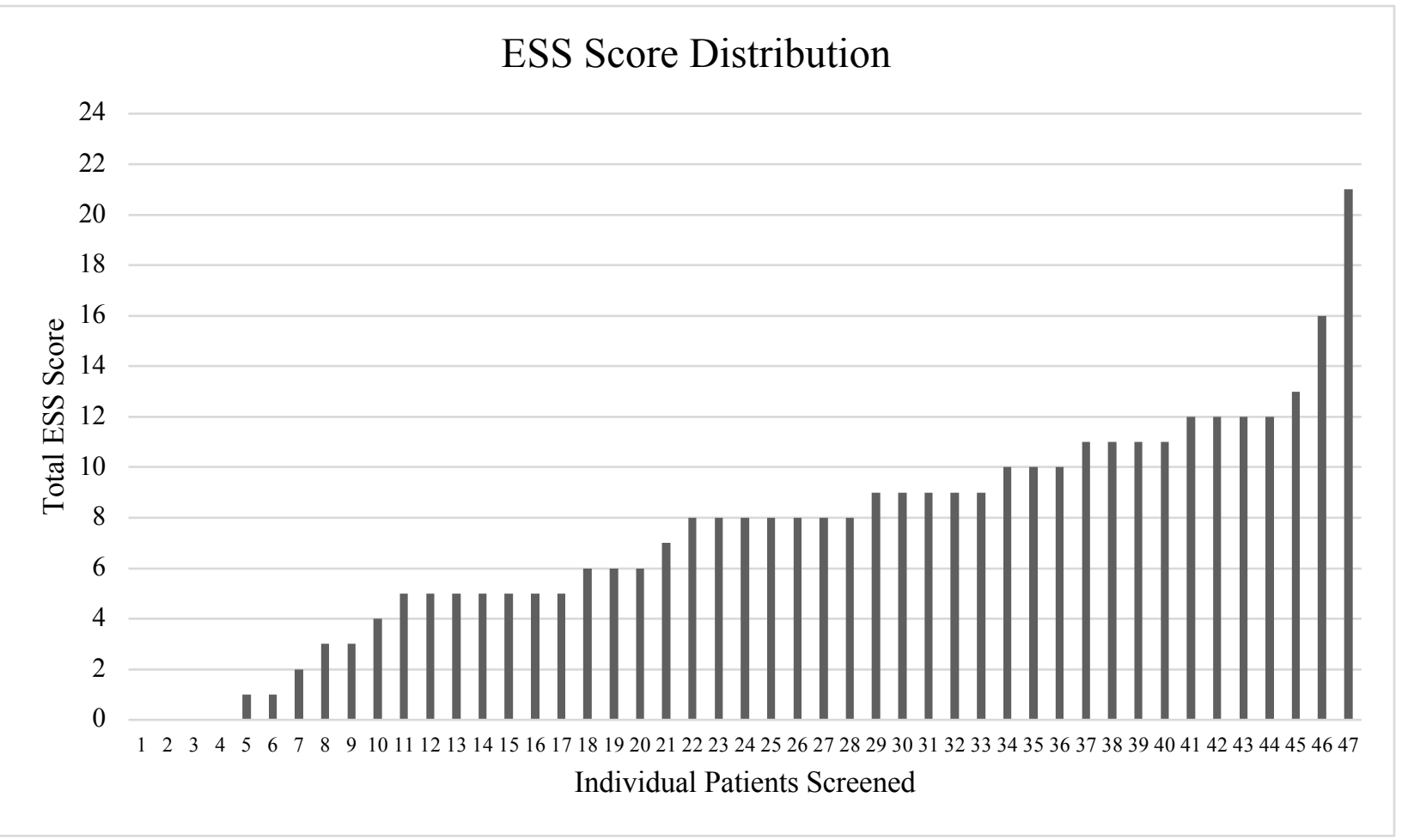

Figure 4. Epworth Sleepiness Scale risk stratification

\begin{tabular}{|c|c|c|}
\hline Line Item & Explanation of Cost & Total Estimated Cost \\
\hline $\begin{array}{c}\text { Screening patients without } \\
\text { OSA diagnosis } \\
10 \text { hours }\end{array}$ & $\begin{array}{c}\text { DNP student }=\text { Free } \\
\text { Estimated cost to sustain at } \\
\$ 35 / \text { hour }\end{array}$ & $\$ 350$ \\
\hline $\begin{array}{c}\text { Training } \\
1 \text { hour }\end{array}$ & $\begin{array}{c}\text { 1x DNP student }=\text { Free } \\
4 \times \text { RN } \$ 35 / \text { hour }\end{array}$ & $\$ 140$ \\
\hline Cost of ESS Tool & $\begin{array}{c}\text { Existing license for use in VA } \\
\text { EHR }\end{array}$ & $\$ 0$ \\
\hline Total Estimated Project Cost & $\begin{array}{l}\text { Total cost - training, tool cost } \\
\text { and } \\
\text { cost to sustain intervention }\end{array}$ & $\begin{array}{l}\text { Total duration of intervention } \\
\text { (30 days) } \\
=\$ 490 \\
\text { Sustained intervention } \\
\text { (monthly) } \\
=\$ 350\end{array}$ \\
\hline
\end{tabular}

Figure 5. Intervention costs 


\begin{tabular}{|c|c|c|c|}
\hline$\underline{\text { Items }}$ & Cost & Explanation & $\underline{\text { Savings }}$ \\
\hline $\begin{array}{c}\text { Cost for OSA related } \\
\text { DM and HTN }\end{array}$ & & & \\
\hline $\begin{array}{l}\text { hospitalizations } \\
\text { annually } \\
(\$ 11,000,000 * 2.1 \% \\
\text { for HTN and } 2.9 \% \text { for } \\
\text { DM })\end{array}$ & $\$ 660,000$ & $\begin{array}{c}\$ 660,000 * 7 \% \text { for } \\
\text { representative Veteran } \\
\text { population }\end{array}$ & $\begin{array}{l}\$ 46,200 \text { potential } \\
\text { savings annually } \\
\text { (\$3,850 monthly) }\end{array}$ \\
\hline Intervention (Initial) & $\$ 490$ & $\$ 3,850 / \$ 490$ & $\begin{array}{c}\$ 7.8 \text { saved for each } \$ 1 \\
\text { spent }\end{array}$ \\
\hline $\begin{array}{l}\text { Intervention } \\
\text { (Sustained) }\end{array}$ & $\$ 350$ & $\$ 3,850 / \$ 350$ & $\begin{array}{c}\$ 11 \text { saved for each } \$ 1 \\
\text { spent }\end{array}$ \\
\hline
\end{tabular}

Figure 6. Cost benefit analysis (per month) 